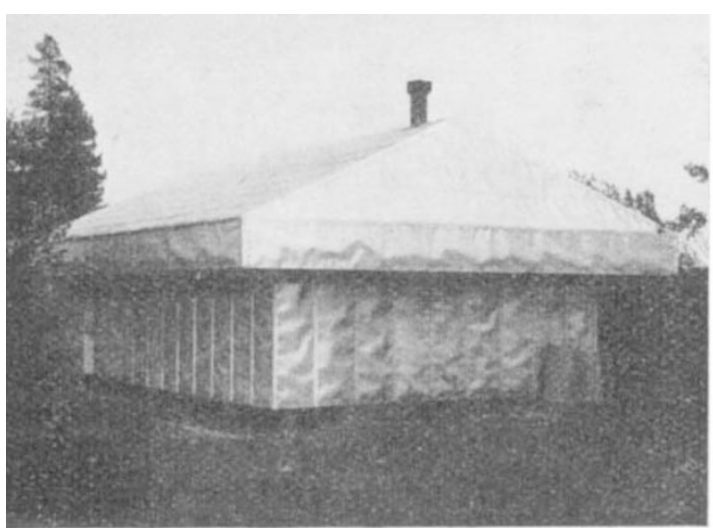

Fig 3

provisory building in 1948. Two registration houses are now accommodating two special-purpose cosmicray telescopes besides two 'international cubics'. A widely soparated set of counters for investigation of extensive air showers is under construction. In 1951 four seismographs were installed in a special building at the Observatory.

The establishment of a first-class geophysical observatory near Kiruna was proposed by the Royal Swedish Academy of Science more than a decade ago. Its planning has been in the hands of a special Board appointed by the Royal Swedish Academy of Science. The members of this Board are: Senator Dr. R. Sandler (chairman); Dr. R. Sievert (vice-chairman), professor of radiophysics, Royal Caroline Medicalsurgical Institute, Stockholm ; Dr. H. Alfvén, professor of electronics, Royal Technical University of Stockholm ; Dr. N. Ambolt, director of the Section of Terrestrial Magnetism, Royal Board of Shipping

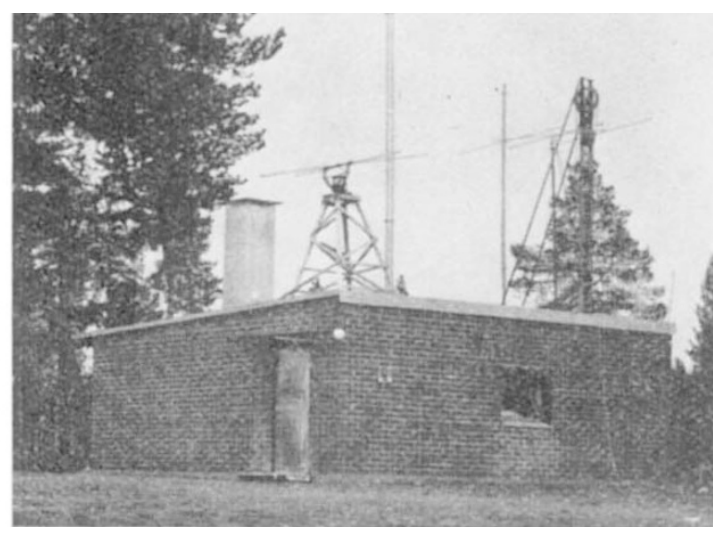

Fig. 4

and Navigation, Stockholm ; Dr. R. Florin, professor of botany, University of Stockholn; Dr. B. Hanström, professor of zoology, University of Lund; Dr. E. Hulthén, professor of physies, University of Stockholm ; Dr. H. Köhler, professor (emeritus) of meteorology, Universitv of Upsala; Dr. B. Lindblad, professor of astronomy, University of Stockholm, president of the Swedish Natural Science Research Council; Dr. H. Norinder, emeritus professor of electrophysics with special application to atmospheric discharges, University of Upsala; Dr. A. Nyberg, director-in-chief, Swedish Meteorological and Hydrological Institute, Stockholm.

We welcome suitable research proposals, particularly where appropriate financial support can also be provided. All proposals by those interested for co-operative activities should be addressed to the Director, Kiruna Geophysical Observatory, Kiruna, Sweden.

\title{
THE BIOLOGY OF CANCER
}

$\mathrm{T}$ HERE has been an increasing awareness in recont years among those engaged in research of a need for a more fundamental biological approach to the problem of cancer. The general study of viruses was, at one time, looked upon as a pathological problem; but it is now recognized as of importance both in its general biological aspects and in the elucidation of the microstructure of cellular systems. It was therefore refreshing to find this view reflected in a whole-day discussion on "Biological Aspects of Cancer" held by Section D (Zoology) of the British Association in Dublin on September 6. Prof. Rogers Brambell, the chairman for the morning session, regretted the tendency to separate the study of cancer from the general framework of zoology, and emphasized the close relationship between protein synthesis and pathological changes within the cell. Prof. A. Haddow (Chester Beatty Research Institute), who opened the discussion, stressed the fundamental similarity between embryonic and cancer cells, and the need for more information concerning the development of specific enzymes during embryonic development and differentiation.

It was unfortunate that Dr. Julian Huxley was prevented by illness from reading his paper. In some excellent recent reviews, he has stressed the importance of considering the zoological aspects of the problem. He looks upon tumours as constituting a separate major phylum of obligatory parasites, originating and usually perishing with the host, with a way of life most fruitfully considered in the general biological concepts of adaptation, evolution, popula. tion dynamics and the like.

Here it will be convenient to consider three aspects of the problem discussed at the meeting, namely, growth mechanisms and their regulation, population dynamics of tumours and the nature of the intra. cellular transformation giving rise to the manifold properties of cancer cells.

The nature of the environmental controls, by which a healthy organism prevails upon its many cells to adopt and maintain a sociable way of life and which the cancer cells have to overcome, was tackled by several speakers. A knowledge of how these controls are effected remains a major problem of current biology, but there is little doubt that chemical sub. stances, humoral factors, pass from organ to organ influencing both the function and size of the organs. Prof. E. S. Horning (Chester Beatty Research Institute) described the two types of cancer which arise 
spontaneously in both animals and man. One type comprises a group of malignant tumours the growth of which can be controlled temporarily by an alteration of the hormonal environment of the body in which they develop. Cancers which respond to this kind of therapy are called hormone-dependent tumours. They are often, but not always, caused by a hormonal imbalance, and are dependent upon a particular hormone or combination of hormones for their sus. tained growth. Many of these tumoura in both animals and man regress once the hormonal stimulus is withdrawn. Prof. Horning went on to describe tumours in the hamster induced by prolonged treatment with the synthetic female sex hormone stilbcestrol. These are an interesting example of a hormone-dependent lesion as they grow, when transplanted under the skin of hamsters, only if they have been pre-treated with oestrogen. These particular tumours have been growing as serial transplants for more than four years, and still remain dependent for their growth on hormones.

An example was also given of the induction of a prostate hormone-dependent tumour in the mouse without employing the direct intervention of a hormonal agent. This prostatic cancer was produced by treatment with one of the carcinogenic hydrocarbons, namely, 20-methylcholanthrene. Many of these primary prostatic tumours were glandular cell carcinomas, and were found to be dependent upon androgens for their maintenance as transplants. During serial transplantations, all these tumours eventually underwent squamous differentiation, and they then became independent of the male sex hormone for their sustained growth as grafts. The dividing line between the hormone-dependent tumours and those which are completely autonomous is not completely rigid. A certain number of dependent tumours, like the mouse prostate carcinoma, transform into autonomous variants, indicating some progressive type of cellular transformation.

In addition to cellular proliferation, tumours may be characterized by their degree of de-differentiation. Differentiation is the process whereby the potentialities of the cells become restricted from the totipotential state of the fertilized egg to the decreased potential state of a muscle cell, red blood cell or cornified cell. Dr. A. Glücksmann (Strangeways Research Laboratory, Cambridge) pointed out that in normal growths, as in epithelium, there is a balance between rate of growth and rate of differentiation. In the development of cancers, this balance may be altered in rate, quality, uniformity of progress and in the type of differentiation impaired. Such changes can arise independently of each other. As in the case of proliferation of cells, changes in differentiation can be brought about by extrinsic environmental conditions, such as hormonal factors operating on the normal or slightly impaired potentialities of the cancer cell.

Another type of change in the cell itself is an alteration of its surface properties. Evidence is accumulating that cell movement, for example, and possibly cell division, are influenced by contact with other cells. Dr. Abercrombie discussed the mechanism of cell movement, which is a property, like that of division, latent in most cells, but which becomes manifest again only when the environmental conditions alter, as in wounds or when cells are transferred to a culture medium in vitro. This work has been considerably assisted by the technique of interferencemicroseope cinematography, which reveals the dynamics of cell movements very clearly. The movements of fibroblasts and perhaps of other cells are associated with peripheral undulations of the cell membrane. When this undulating surface touches another fibroblast, the activity ceases at the point of contact and an adhesion may be formed. These adhesions occur in the phenomenon of inhibition by contact described by Abercrombie and Heaysman. If the cell is in contact with a sufficient number of surfaces its entire membrane becomes quiescent and movement ceases, as is the case in an established tissue. The discovery that cancer cells are not subject to contact inhibition may be of far-reaching impor. tance. In mixed cultures cancer cells may be seen to move freely over the surfaces of normal cells, which. are themselves held motionless, owing to the effects of contact inhibition. It would seem that we have here one of the secrets of the 'success' of the cancer cell. That contact inhibition is a potent factor in tissue construction would seem obvious; in gaining independence from its operation the cancer cell has clearly made the important step towards autonomy and its ability to invade normal tissues can also be understood on this basis.

The films indicate that sarcoma cells in culture do not form permanent adhesions between their undulat. ing membranes. This suggests an alteration in the surface properties of cancer cells, a point of view which has long been held by Coman, who has demonstrated by direct manipulation that cancer cells are less sticky than normal cells. Cancer cells have also recently been found by Ambrose and his co-workers to carry a higher negative electrical charge than homologous normal cells. Clearly these properties are linked, but until more is known of what happens when one cell touches another and what makes some cells stick together and others not, the relationship between cell adhesion, contact inhibition and tissue construction can only be guessed.

The fine details of the structure of the cell membrane and of the contacts themselves are now within the province of the electron microscopist. Owing to the recent technical advances allowing the cutting of sections thin enough for electron microscopy, there has been a rapid growth in our knowledge of the fine structure of cells. Dr. E. H. Mercer (Chester Beatty Research Institute) described how these techniques are being adapted to the problem of cell contact and cell adhesion. By the choice of certain simple cell systems, such as the mammalian epidermis and a species of social amoba, in which the cells progress from a relatively free-living condition to form welldifferentiated structures, the behaviour of the cell membranes can be followed. The external membrane of the amcere at high resolution is resolved into a lamellar structure about $60 \mathrm{~A}$. thick, consisting of two dense surfaces enclosing a less dense layer. In some instances, the membranes of other cells can also be resolved in this way. The structure may correspond to the model of two protein layers spread over a lipoid-like layer proposed by Danielli. When two cells meet, the plasma membranes flatten out, implying that the cells 'wet' each other, but the dense layers of the membranes never actually meet. They remain separated by a thin layer of less-dense material, 150-200 A. thick. This suggests the presence of some extracellular cementing substance outside the plasma membrane. 
Such a material should obviously play a key part in tissue formation and maintenance and will affect many of the surface properties of cells. The possibility of a loss in quantity or perhaps quality of this cement is suggested by electron micrographs of anaplastic tumours the membranes of which show poor attachment. In more differentiated tumours, however, the morphology of the contacts still appears normal.

The nature of the cell surface was further discussed by Dr. R. J. Goldacre and Drs. G. C. and D. M. Easty. Dr. Groldacre (Chester Beatty Research Institute) pointed out that many of the fundamental aspects of cell physiology can be studied with any type of living cell. The large Amoba proteus is a convenient test organism in which cellular changes can be seen much more easily by eye than in the smaller tissue cells. Certain carcinogens such as nitrogen mustards and diepoxides can be seen to have a direct tanning effect on the membrane, preventing the animal from retracting its pseudopods. He showed a film which indicated that at least a part of protein synthesis takes place at the cell membrane. Since there is considerable experimental evidence that the cell membrane is altered in cancer cells, this could account for an altered type of protein synthesis. Another possibility is that membranes are concerned in the transfer of information from one cell to another, and that this transfor could be impaired were the membranes modified.

Dr. and Mrs. G. C. Easty (Chester Beatty Research Institute) were concerned to show, by means of specific surface antisera, that the surfaces of the cells of different organs were different antigenically and that, in a particular transplanted tumour they examined, the tumour cells differed from normal cells. An antiserum to a cell can produce three offects : precipitation of cells, aggregation and a change in permeability of the membrane. This last effect can be particularly well seen in the interference microscope because it is associated with a sudden passage of cytoplasmic protein from the cell, which can be observed as a dramatic change of colour in the interference image. An antiserum against ascites cells contains antibodies against a variety of cells, but is purified by adsorbing against normal cells and can be obtained with an increased degree of specificity against the cancer cells. Films show that this antiserum reacts specifically with the membrane of cancer cells in the presence of other normal cells. This result raises the possibility of a tumour-specific antiserum in therapeutics, but requires further investigation using primary tumours. Dr. Easty also showed that in some tumours the antiserum reacts on some cells without affecting others, indicating the presence of a heterogeneous population.

In general biological terms we may now ask our. selves what is the origin of the new species of cells? Probably the new cells are mutants less under the control of those environmental factors which are able to repress the spontaneous tendency of the unmutatod stock to divide. Mutants, capable of complete autonomy, probably do not arise in a single step. The initial variants may bo supposed to have a slightly enhanced autonomy, but can probably only increase in a localized host environment where the normal powers of control are impaired by the action of some promoting condition. In such conditions, natural seloction operating on a mixed population of altered cells may be relied upon to select a strain of increasing autonomy. Prof. P. C. Koller (Chester Beatty Research Institute) showed a selection of cell smears illustrating chromosomal variations. These provide visual proof of the fact of chromosomal change in cancer cells and also of the existence of numerous chromosomal variants in the cell population, implying the presence of mutant forms. This can clearly be seen in tho diversity of chromosome number. These changes are brought about largely by abnormal mitosis, such as lack of synchronization of nuclear and cytoplasmic division. Multiple spindles, operating on a mass of chromosomes, and the occurrence of anaphase bridges also lead to variations in the nuclear size of daughter cells. Cellular polymorphism provides a basis for the operation of selection as the tumour develops, invades and occupies new situations. This may be shown by changes in the number of chromosomes between the primary and secondary tumours. Certain cell variants may also be favoured during therapeutic treatment when the cells are subject to radiation or chemotherapeutic agents. Prof. G. Klein of Sweden has also studied this problem using transplantation techniques, and has produced a number of strains from a single tumour which show differences in degrees of invasiveness and other biological behaviour.

The origin of the new types of cell, in chemical carcinogenesis, was discussed by Prof. Haddow. He pointed out that chemical carcinogenesis provides a direct experimental approach to this problem. Since the discovery of the carcinogenic hydrocarbons a number of comparatively simple chemical substances have been discovered which can induce cancer, for example, the nitrogen mustards, epoxides, and ethyleneimines. Their possible reactions with biological material are limited; the nitrogen mustards, for example, operate by alkylation. Those which are of a bifunctional nature are far more active biologically than monofunctional compounds. Another advantage of these compounds, from the point of view of experimental observation, is that thoy have a rapid effect upon the cell. A number of direct cytological changes are produced by these agents : fragmentation of chromosomes, anaphase bridges, interference with chromosome duplication, and formation of micronuclei. Such effects are also produced by radiation, and the substances are therefore called radiomimetic agents. The direct effect of these agents upon nucleic acid in vitro is known, and it appears distinctly possible that their biological reaction is with this component of the cell. They are found to have a marked mutagenic action in Drosophila. Although some alteration in the genetic constitution of the cell may bo involved in the malignant change, control of growth in terms of cellular dynamies probably depends upon enzyme systems. Xanthoptorin, for example, will produce a wave of mitosis in kidney tubulos. Xanthine oxidase appears to be the onzyme which controls the level of xanthopterin in normal tissues, and there is some evidence that its concentration is reduced in some tumours.

There were some rather obvious omissions from the programme in Dublin. Little was said about the virus theory of cancer, although a number of mammalian tumours have recently been shown to $b e$ of viral origin. Nor was the immunological response of the host to a growing tumour examined. But it was obviously impossible to cover all these aspects in a single day. 